\title{
Quenched disorder formulation of the pseudo-gap problem
}

\author{
A. Posazhennikova ${ }^{1, *}$ and P. Coleman ${ }^{2}$ \\ 1 Laboratorium voor Vaste-Stoffysica en Magnetisme, \\ Katholieke Universiteit Leuven, Celestijnenlaan 200 D, B-3001 Leuven, Belgium \\ ${ }^{2}$ Center for Materials Theory, Department of Physics and Astronomy, \\ Rutgers University, Piscataway, NJ 08854, USA
}

\begin{abstract}
The problem of pseudo-gap formation in an electronic system, induced by the fluctuations of the order parameter is revisited. We make the observation that a large class of current theories are theoretically equivalent to averaging the Free energy of the pseudo-gap system over quencheddisordered distribution of the order parameter. We examine the cases of both infinite and finite correlation length, showing how the interplay of pseudo-gap formation and superconductivity can be treated in this approach.
\end{abstract}

*Email anna@tkm.physik.uni-karlsruhe.de 


\section{INTRODUCTION}

The discovery of a marked suppression in the density of states of the underdoped cuprate superconductors above the critical temperature has led to a revival of interest in the idea of a pseudo-gap. This concept was originally introduced by Lee, Rice and Anderson [1] to explain the suppression of electron density of states associated with order parameter fluctuations near a charge density wave instability. According to one school of thought, a similar mechanism may drive the formation of the pseudo-gap in the underdoped cuprates(see reviews

[2, 3, 4]). Many candidate fluctuating order parameters, such as antiferromagnetism [5], charge density wave [6] and pre-formed Cooper pairs have been proposed [7].

Pseudo-gaps are likely to be a widespread feature of correlated electron systems lying close to an instability that gaps part of the Fermi surface, and they have been observed in a wide variety of nested and low dimensional strongly correlated electron materials, including one dimensional charge density wave systems[8], vanadium doped Chromium [9], colossal magneto-resistance compounds [10] and strontium-calcium and strontium-barium ruthenates [1]].

The fluctuation gap model (FGM), based on the idea of the pseudo-gap emergence due to fluctuating order parameter was pioneered in 1]. Later on it was shown by Sadovskii[12, 13] how the problem of nested electrons moving in a critically fluctuating order parameter field could be solved exactly. Interest in this model was revived with the discovery of the pseudogap in cuprates, the physical origin of which is still controversial. Schmalian et al [14] extended the 1D model to higher dimensions and nonzero spin. Tchernyshyov [15] later questioned the exact solvability of the model for finite correlation lengths and pointed out a previously unnoticed mistake in the Sadovskii result. Couple of years ago Millis and Monien[16] performed a careful study of different approximations, explaining for pseudogaps, also by Bartosch and Kopietz [17] the exact numerical calculation of the density of states in FGM was carried out. They demonstrated [16, 17] that Sadovskii approach is in fact a good approximation and the terms, omitted in his model produce negligible corrections. In a related development, Kopietz et al [19] presented the exactly solvable model of extended FGM, where fluctuations of both phase and amplitude of the order parameter have been taken into account. The important issues of crossover from Gaussian to non-Gaussian order parameter fluctuations in one-dimensional Peierls systems have been recently discussed by 
Monien [20].

A key idea of the Lee, Rice and Anderson paper [1] is that order parameter fluctuations can be regarded as classical degrees of freedom with time-independent correlation functions. This basic assumption threads through most of the subsequent developments during past twenty years. This paper revisits this basic idea, making the observation that a large class of current theories are theoretically equivalent to averaging the Free energy of the pseudo-gap system over a quenched-disordered distribution of the order parameter. The intuitive equivalence between the slow critical fluctuations responsible for pseudo-gap formation and a quenched random potential was certainly known to several authors in the past 1, 21, 22, 23, 24]. In this paper, we vocalize this equivalence and set it in a formal framework. Using the idea we show how the Sadovskii model of pseudo-gap formation can be formulated in terms of a quenched average free energy. To illustrate the utility of this method, we develop a toy model for the interplay of pseudo-gap formation and weak impurity scattering with d-wave superconductivity. Our results may be of interest to recent experiments on the cuprate superconductors.

\section{CLASSICAL INTERACTIONS IMPLY QUENCHED DISORDER}

One of the central assumptions of a large class of pseudo-gap models, is that the orderparameter fluctuations which scatter electrons to form the pseudo-gap are so slow that they can be considered to be infinitely retarded. Such a state of affairs could come about in a variety of ways. The most pragmatic point-of-view, is that the characteristic relaxation timescale of the fluctuations $\tau_{O P}$ is not actually infinite, but may be treated as such because it is much greater than the inelastic scattering rate of the electrons $\tau_{e}$

$$
\tau_{O P}>>\tau_{e}
$$

The result of this condition, is that electrons perceive the order parameter as a frozen degree of freedom, that may be treated as a classical variable.

We now need to consider the interactions between the electrons induced by these classical order parameter fluctuations. In a Feynman diagram, the net interaction induced by these fluctuations has a number of important features (Fig. 1.):

- It transfers no energy, and is thus infinitely retarded. (Fig. 1 (a)) 
- Polarization bubble insertions into the effective interaction are absent, since these have already been taken into account in forming the effective interaction (Fig. 1(b)).

- Interactions between the classical fluctuations may be ignored. This is a useful, assumption, for it leads to non-interacting Gaussian fluctuations and permits the summation of a large class of diagrams. Unfortunately, it can not really be justified near a critical point, where non-linear interactions between classical fluctuations are almost always relevant. This is without doubt a major weakness of the current class of theories, and one we shall return to in our final discussion.

(a)

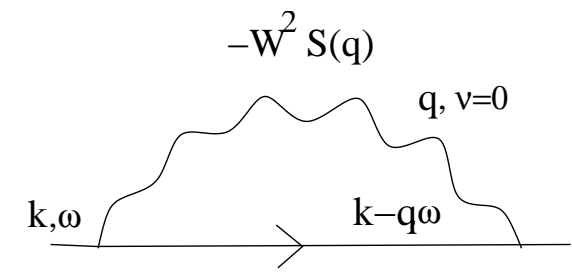

(b)

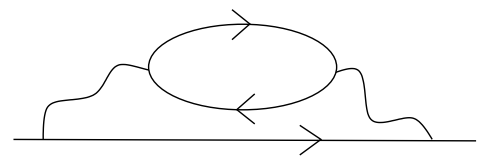

(c)

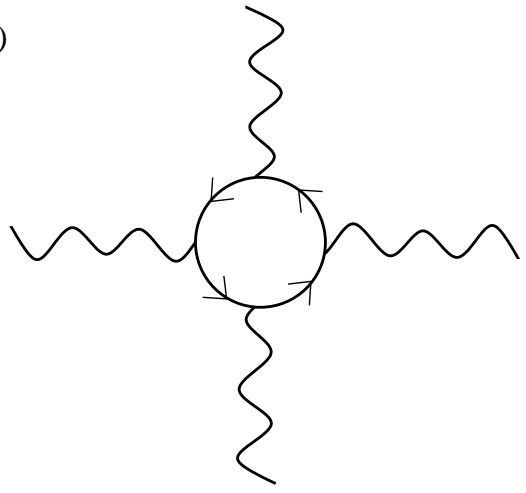

FIG. 1: (a) Classical fluctuations induce an interaction $V_{\text {eff }}=-W^{2} S(\vec{q})$ on a single line. (b) Fermion loop insertions into the interaction line are neglected which means that the average over the classical fluctuations is a quenched average. (c) By carrying out a quenched average, we also eliminate interactions between fluctuations of the form shown here.

The first feature can be incorporated into a model by introducing a coupling into the Hamiltonian

$$
H[\phi]=H_{o}+W \sum_{\vec{q}} \rho_{\vec{q}} \phi_{-\vec{q}} .
$$

In this paper we will imagine that $H_{0}$ is a Hamiltonian in which effects of interaction have been taken into account by a mean-field approximation- so that for instance, $H_{0}$ might be 
a BCS Hamiltonian with explicit pairing terms. In (11) $\rho_{\vec{q}}=\sum_{\vec{k}} c_{\vec{k}-\vec{q}}^{\dagger} c_{\vec{k}}$ is the density of electrons at momentum $\vec{q}$ and $\phi_{\vec{q}}$ is a purely classical field representing the order parameter fluctuations. This quantity is to be averaged over the distribution

$$
P[\phi]=\exp \left[-\beta \sum \frac{\left|\phi_{\vec{q}}\right|^{2}}{2 S(\vec{q})}\right]
$$

and it thus gives rise to an effective, and infinitely retarded (attractive) interaction of the form

$$
V_{e f f}(\vec{q})=-W^{2} S(\vec{q})
$$

At first sight, it might appear sufficient average the partition function over $P[\phi]$, i.e

$$
\begin{aligned}
Z & =\int P[\phi] Z[\phi] \\
Z[\phi] & =\operatorname{Tr}\left[e^{-\beta H[\phi]}\right] .
\end{aligned}
$$

This is an annealed average. However, such a scheme allows fermion loop renormalizations and non-linear interactions to develop within the interaction lines as illustrated in Fig. 1 (b). These terms are to be dropped, under the assumptions mentioned above. To ensure this, we must carry out a quenched average over the classical field, which means that we average the Free energy, rather than the partition function

$$
F=-T \int P[\phi] \log Z[\phi]
$$

This procedure is implicit to the approach of Sadovskii, and subsequent developments, by the selection of Feynman diagrams containing a single Fermion line. One way to implement the quenched average is to use the $N \rightarrow 0$ trick,

$$
F=-T \lim _{N \rightarrow 0} \int P[\phi] \frac{1}{N}\left(Z[\phi]^{N}-1\right) .
$$

The term containing $Z[\phi]^{N}$ can be considered as the partition function of $\mathrm{N}$ identical replicas in the frozen classical field $\phi$. Formally, this is accomplished by introducing $N$ replicas of the fermion fields, labeled by the index $\lambda \in[1, N]$. The partition function is then given by

$$
Z^{N}[\phi]=\operatorname{Tr}\left[e^{-\beta H^{(N)}[\phi]}\right]
$$

where

$$
H^{(N)}[\phi]=\sum_{\lambda=1, N}\left(H_{o}^{\lambda}+W \sum_{\vec{q}} \rho_{\vec{q}}^{\lambda} \phi_{-\vec{q}}\right) .
$$


is the Hamiltonian of $N$ identical replicas coupled to a single classical field. Diagrammatic contributions to $Z[\phi]^{N}$ containing $n$ fermion loops, scale as $O\left(N^{n}\right)$, so that in the $N \rightarrow 0$ limit, the only terms surviving in the diagrammatic expansion of the Free energy, are those with a single fermion loop, as follows

$$
\Delta F=-T \lim _{N \rightarrow 0}\left[\frac{1}{N}(\overbrace{\sum_{\lambda}+\lambda_{\lambda}+\ldots}^{O(N)} \overbrace{\lambda+\lambda_{\lambda}^{\lambda}+\ldots}^{O\left(N^{2}\right)}],\right.
$$

where $\Delta F=F_{I}-F_{0}$ is the change in the Free energy due to turning on the interactions, Thus the only terms which survive the $N \rightarrow 0$ limit are those with a single fermion line or loop [26]. In this way, all RPA renormalizations and non-linear interactions between the fluctuation fields are eliminated, as follows,

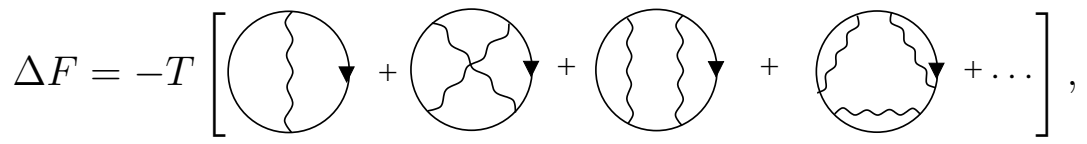

where the replica index has been eliminated from the fermion lines. The advantage of this approach is that we can now start to formulate a theory of the pseudo-gap in the language of an effective Free energy. This becomes particularly useful when considering the case of a superconductor with a pseudo-gap.

\section{EFFECTIVE ACTION IN THE PSEUDO-GAP MODEL WITH INFINITE CORRELATION LENGTH}

To illustrate the above approach, we consider a two-dimensional electronic system with a nested Fermi surface spanned by the commensurate wavevector $\vec{Q}=(\pi, \pi)$ as illustrated in Fig. 2. Fluctuations of the underlying order parameter are governed by a correlation function of the form

$$
S(\vec{q})=\frac{1}{\pi^{2}} \frac{\xi^{-1}}{\left(q_{x}-Q_{x}\right)^{2}+\xi^{-2}} \frac{\xi^{-1}}{\left(q_{y}-Q_{y}\right)^{2}+\xi^{-2}}
$$

fluctuations couple the nested Fermi surfaces via the effective interaction $V_{\text {eff }}=-W^{2} S(\vec{q})$. It was shown [25] that in the limit $\xi \rightarrow \infty$ the model under consideration can be solved exactly and the effective interaction takes the oversimplified form

$$
V_{e f f}(\vec{q})=-W^{2}\left\{\delta\left(q_{x}-\pi\right) \delta\left(q_{y}-\pi\right)\right\}
$$




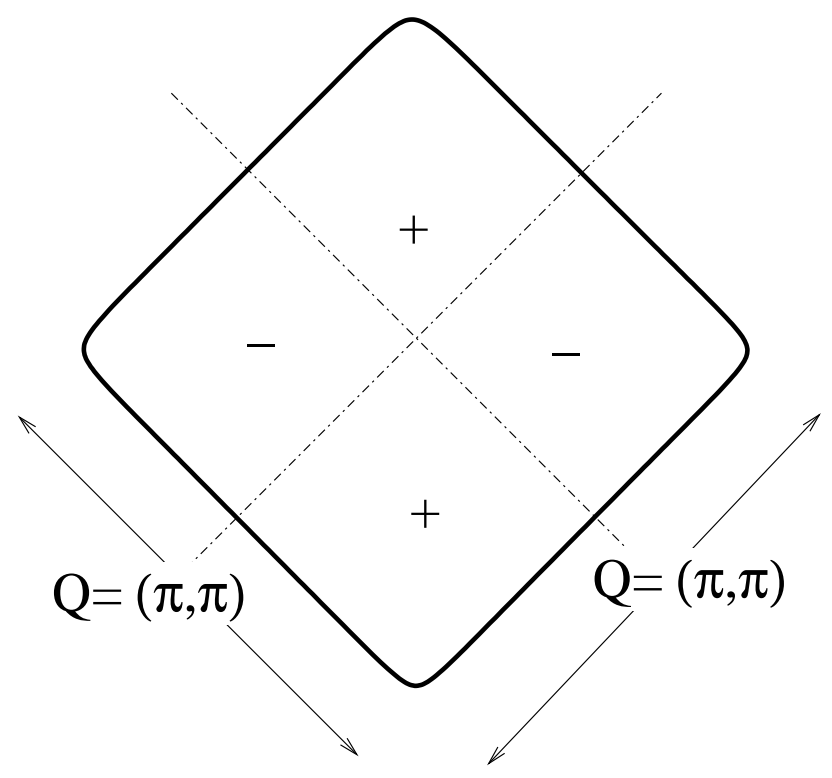

FIG. 2: Nested Fermi surface with commensurate fluctuations considered in the toy model of this paper.

In this paper, we will compliment the earlier work 25] by showing how the diagrams for the Free energy may be completely summed in this limit. The class of models we shall discuss take the form

$$
H=H_{0}+H_{I}
$$

where $H_{0}$ is a Hamiltonian which does not scatter between the nested Fermi surfaces and $H_{I}$ contains the coupling to the density fluctuation modes. We begin by considering the simple model where

$$
\begin{aligned}
& H_{0}=\sum_{\vec{k}} \epsilon_{\vec{k}} c_{\vec{k} \sigma}^{\dagger} c_{\vec{k} \sigma} \\
& H_{I}=W \sum_{\vec{k}, \vec{q}} c_{\vec{k}+\vec{q}}^{\dagger} c_{\vec{k}} \phi_{\vec{q}},
\end{aligned}
$$

where $\phi_{\vec{q}}$ is a classical field described by quenched average over the probability distribution $P[\phi]$ given above. If we denote the effective action by a wavy line, then the Feynman diagrams for the expansion of the Free energy are 


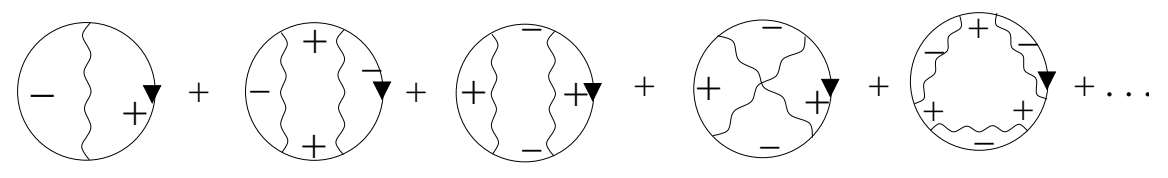

Here we have implicitly taken the $N \rightarrow 0$ limit of (4), thereby eliminating all polarization renormalizations of the interaction lines. We have used the pseudo-momentum labels " - " and "+" to denote electrons lying on the "left" or "right"-hand side of the nested Fermi surface. An essential feature of our derivation is that the pseudo-momentum alternates between scattering events. (For incommensurate fluctuations, this requirement eliminates some of the diagrams such as the fourth diagram shown above). In the limit of an infinite correlation length, each interaction line can effectively be replaced by a static modulated scattering potential which scatters at the nesting wavevectors. Consider the simplest $W^{2}$ diagram. In the limit of infinite correlation length, the interaction can be replaced by a delta function in momentum, so that

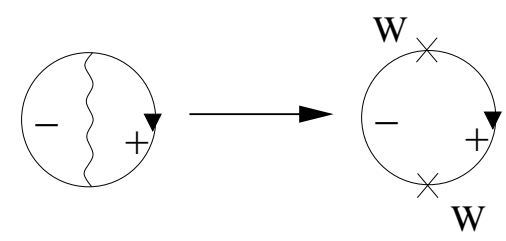

where we have represented the vertex of the static scattering potential by a "cross". When we come to consider higher order diagrams, each term of a given order gives rise to the same contribution, however, we must be careful to take into account symmetry factors. Let the number of closed diagrams of order $W^{2 n}$ be $\alpha_{n}$, then

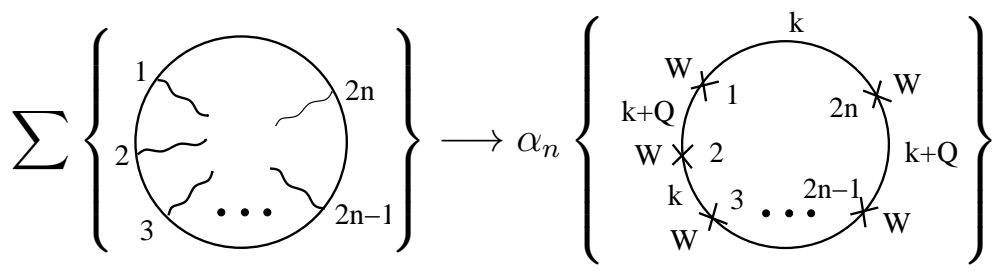

Around the perimeter of each diagram of this order, there are $n$ propagators of both "-" and "+" pseudo-momentum. If we differentiate the sum of all $2 n$th order diagrams with respect to $G_{\vec{k}}$, we generate the $2 n$th order self-energy diagram, as follows 


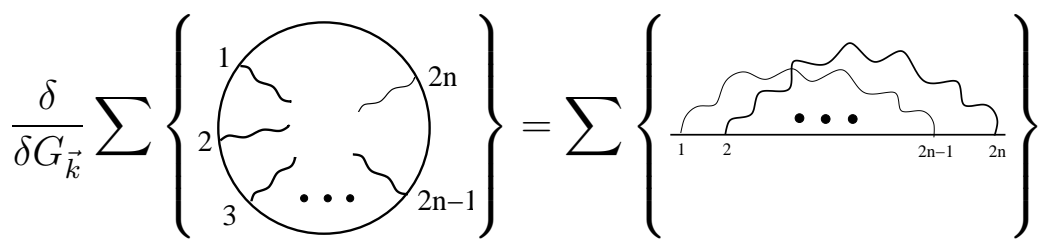

But in the limit of infinite correlation length, this is equal to

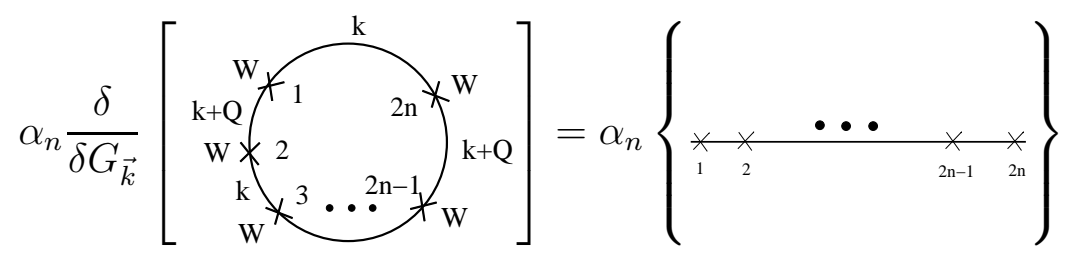

Now the number of $2 n$th order self energy diagrams is given by the number of ways of connecting $\mathrm{n}$ interaction propagators to $2 \mathrm{n}$ interaction vertices so that

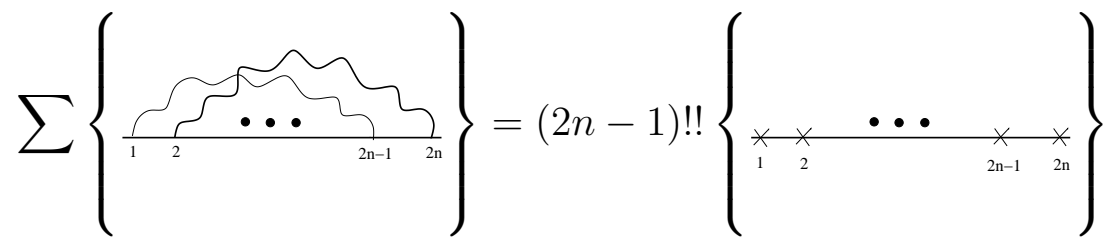

enabling us to identify $\alpha_{n}=(2 n-1)$ !!. Using the relation

$$
(2 n-1) ! !=\frac{2^{n}}{\sqrt{\pi}}\left(n-\frac{1}{2}\right) !
$$

where we denote $\left(x-\frac{1}{2}\right) ! \equiv \Gamma\left(x+\frac{1}{2}\right)$, we can absorb the factor $(2 n-1) ! !$ into an integral over a Gaussian distribution of gap sizes as follows

$$
W^{2 n}(2 n-1) ! !=\frac{1}{\sqrt{2 \pi}} \int_{-\infty}^{\infty} d \zeta e^{-\zeta^{2} / 2}(\zeta W)^{2 n},
$$

in other words

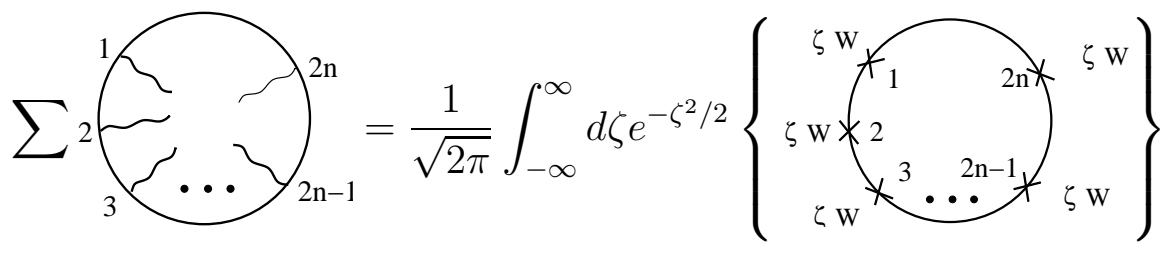


Remarkably- all that is left of the average over the classical fields is a residual distribution over pseudo-gap sizes. In other words, in the limit of an infinite correlation length, the quenched average over the distribution of scattering potentials is replaced by a single average over a Gaussian distribution of scattering strengths. The quenched averaged Free energy is then given by

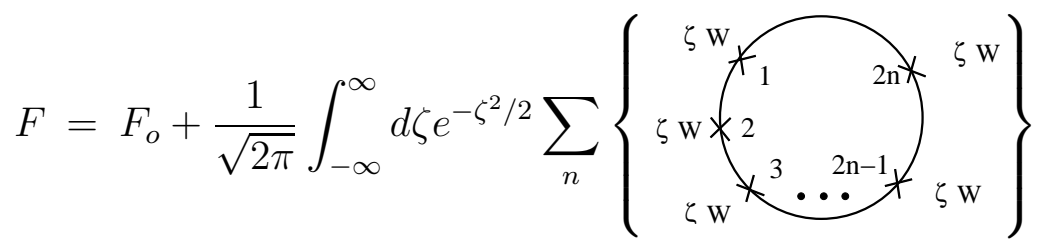

where $F_{o}=-T \sum_{\vec{k}, n} \ln \left[-G_{o}^{-1}\left(\vec{k}, i \omega_{n}\right)\right]$ is the Free energy of the "non-interacting" system. Combining both terms, we then obtain

$$
F=\frac{1}{\sqrt{2 \pi}} \int_{-\infty}^{\infty} d \zeta e^{-\zeta^{2} / 2} F[\zeta, W]
$$

where

$$
F[\zeta, W]=-T \sum_{n, \vec{k} \in \frac{1}{2} \mathrm{BZ}} \operatorname{Tr} \ln \left(\begin{array}{cc}
-G_{o}^{-1}\left(\vec{k}-\vec{Q} / 2, i \omega_{n}\right) & \zeta W \\
\zeta W & -G_{o}^{-1}\left(\vec{k}+\vec{Q} / 2, i \omega_{n}\right)
\end{array}\right)
$$

is the Free energy for a gap size $\zeta W$. The most important feature of our derivation above, is the conservation of the pseudo-momentum associated with each nested Fermi surface. This derivation can be easily generalized to include pairing (which conserves momentum ) and a restricted class of disorder scattering which scatters between states on the same nested side of the Fermi surface. We now examine these two cases in detail

\section{A. Pairing in the presence of a pseudo-gap}

To consider pairing, let us now take

$$
H_{0}=\sum_{\vec{k}} \epsilon_{\vec{k}} c_{\vec{k} \sigma}^{\dagger} c_{\vec{k} \sigma}+\sum_{\vec{k}}\left[\Delta \gamma_{\vec{k}} c_{\vec{k} \uparrow}^{\dagger} c_{-\vec{k} \downarrow}^{\dagger}+\text { H.c. }\right]+\frac{|\Delta|^{2}}{g} .
$$

where $\gamma_{\vec{k}}=\cos \left(k_{x}\right)-\cos \left(k_{y}\right)$ is a d-wave order parameter whose nodes bisect the nested Fermi surfaces. We have included an additional term $|\Delta|^{2} / g$ to take account of the BCS 
decoupling of the pairing interaction. To make the model more interesting, we shall consider a pseudo-gap with d-wave symmetry,

$$
H_{I}=W \sum_{\vec{k}, \vec{q}} \gamma_{\vec{k}} c_{\vec{k}+\vec{q}}^{\dagger} c_{\vec{k}} \phi_{\vec{q}}
$$

where $\gamma_{\vec{k}}=\cos \left(k_{x}\right)-\cos \left(k_{y}\right)$ as before. Since the pairing does not change the momentum of particles, the reasoning for a normal state is quickly generalized by using the appropriate Nambu electron propagators. Let us first derive the free energy expression for this case. The mean -field Hamiltonian can be expressed in the following general form

$$
H=\sum_{k \in \frac{1}{2} B z} \Psi_{k}^{+} \mathcal{H} \Psi_{k}+\frac{|\Delta|^{2}}{g}
$$

where

$$
\Psi_{k}=\left(\begin{array}{c}
c_{k+Q / 2 \uparrow} \\
c_{-k-Q / 2 \downarrow}^{+} \\
c_{k-Q / 2 \uparrow} \\
c_{-k+Q / 2 \downarrow}^{+}
\end{array}\right)
$$

and

$$
\mathcal{H}=\left(\begin{array}{cccc}
\epsilon_{k+Q / 2} & \Delta_{k+Q / 2} & W_{k} & 0 \\
\Delta_{k+Q / 2} & -\left(\epsilon_{k+Q / 2}\right) & 0 & -W_{-k} \\
W_{k} & 0 & \epsilon_{k-Q / 2} & \Delta_{k-Q / 2} \\
0 & -W_{-k} & \Delta_{k-Q / 2} & -\epsilon_{-(k-Q / 2)}
\end{array}\right)
$$

where $W_{\kappa}=\sin \kappa_{x}-\sin \kappa_{y}$. Now since $\epsilon_{\kappa-Q / 2}=\epsilon_{-(\kappa+Q / 2)}, \Delta_{\kappa-Q / 2}=-\Delta_{\kappa+Q / 2}$ and $W_{\kappa}=$ $-W_{-\kappa}$, we can write

$$
\mathcal{H}=\left(\begin{array}{cccc}
\epsilon_{\kappa+Q / 2} & \Delta_{\kappa+Q / 2} & W_{\kappa} & 0 \\
\Delta_{\kappa+Q / 2} & -\epsilon_{\kappa+Q / 2} & 0 & +W_{\kappa} \\
W_{\kappa} & 0 & -\epsilon_{\kappa+Q / 2} & -\Delta_{\kappa+Q / 2} \\
0 & W_{\kappa} & -\Delta_{\kappa+Q / 2} & \epsilon_{\kappa+Q / 2}
\end{array}\right)
$$

so that

$$
\operatorname{Tr} \ln \left[-\mathcal{G}^{-1}\left(i \omega_{n}\right)\right]=\ln \operatorname{det}\left[\mathcal{H}-i \omega_{n}\right]=\ln \left[\omega_{n}^{2}+\epsilon_{k+Q / 2}^{2}+\Delta_{k+Q / 2}^{2}+W_{k}^{2}\right]
$$

so that the the expression for the quenched-averaged Free energy takes the form

$$
F=\frac{1}{\sqrt{2 \pi}} \int_{-\infty}^{\infty} d \zeta e^{-\zeta^{2} / 2}\left\{-2 T \sum_{n, k \in \frac{1}{2} \mathrm{BZ}} \ln \left[\omega_{n}^{2}+\epsilon_{k+Q / 2}^{2}+\Delta_{k+Q / 2}^{2}+\left(\zeta W_{k}\right)^{2}\right]+\frac{\Delta^{2}}{g}\right\} .
$$


The gap-equation is provided by the condition that the derivative of the free energy is equal to zero, $\frac{\partial F}{\partial \Delta}=0$, i.e

$$
\frac{1}{g}+\frac{1}{\sqrt{2 \pi}} \int_{-\infty}^{\infty} d \zeta e^{-\zeta^{2} / 2}\left\{-2 T \sum_{n, k \in \frac{1}{2} \mathrm{BZ}} \frac{\gamma_{k}^{2}}{\omega_{n}^{2}+\epsilon_{k}^{2}+\Delta_{k}^{2}+\zeta^{2} W_{k-Q / 2}^{2}}\right\}=0 .
$$

At the critical temperature $T_{c}, \Delta=0$ and we have the transition temperature equation

$$
\frac{1}{g}=\frac{1}{\sqrt{2 \pi}} \int_{-\infty}^{\infty} d \zeta e^{-\zeta^{2} / 2}\left\{2 T \sum_{n, k \in \frac{1}{2} \mathrm{BZ}} \frac{\gamma_{k}^{2}}{\omega_{n}^{2}+\epsilon_{k}^{2}+\zeta^{2} W_{k-Q / 2}^{2}}\right\}
$$

In this way, we have successfully eluded the diagrammatic derivation of the two-particle Green's function [25] and derived the main result in a compact and more general way.

\section{B. Impurity effect}

As a further application of these methods, let us now introduce disorder into the paired pseudo-gap model of the last section. The interplay of disorder and pseudo-gap formation on pairing is of particular interest in the context of underdoped cuprate superconductors. In a recent experimental paper, Tallon et al. [18] have shown that the superconducting transition temperature of $L a-241$ and $(Y, C a)-123$ is much more rapidly suppressed by disorder in underdoped compounds, where a pseudo-gap is present. Tallon et al explained their results in terms of a phenomenological model involving unitary scattering taking place. We now show how the essential features of their discussion can also be obtained by treating the co-existence of pseudo-gap formation and weak non-magnetic impurity scattering.

In the presence of impurity scattering we have to include the additional terms in our Hamiltonian of the form

$$
H^{\prime}=\sum_{j, k k^{\prime} \sigma} e^{-i\left(k-k^{\prime}\right) \cdot \vec{R}_{j}} V_{k k^{\prime}} c_{k \sigma}^{\dagger} c_{k^{\prime} \sigma}
$$

In order to apply the methods of the last section, we make the important assumption that impurity potential $V_{k k^{\prime}}$ does not scatter between different sides of the Fermi surface. Such scattering can still transfer electrons across the node, and thus remains severely pair-breaking. With this simplified assumption, the impurity scattering does not renormalize the strength of the pseudo-gap potential. 
We can write now

$$
F=\frac{1}{\sqrt{2 \pi}} \int_{-\infty}^{\infty} d \zeta e^{-\zeta^{2} / 2} F\left[\zeta, V_{k k^{\prime}}\right] .
$$

In this oversimplified model the effect of impurities reduces just to replacing of thin lines to there "thick", impurity dressed equivalents in the graphic representation for the two-particle Green's function in our model.

Using this result, we can generalize equation (30) to obtain

$$
\frac{1}{g}=T_{c} \sum_{n} \int_{-\infty}^{\infty} \frac{d \zeta}{\sqrt{2 \pi}} e^{-\frac{\zeta^{2}}{2}} \int_{0}^{\infty} \frac{d^{2} k}{(2 \pi)^{2}} \frac{\gamma^{2}(\phi)}{\tilde{\omega}_{n}^{2}+\epsilon_{k}^{2}+\zeta^{2} W^{2}(\phi)},
$$

where $\tilde{\omega}_{n}$ is the usual renormalized Matsubara frequency

$$
\begin{gathered}
\tilde{\omega}_{n}=\omega_{n}+\Gamma \operatorname{sign} \omega_{n} \\
\Gamma=\pi \rho N(0) V_{i m p}^{2},
\end{gathered}
$$

and $\rho$ is the impurity concentration.

Notice that the momentum dependence of $W_{k-Q / 2} \propto \gamma_{k}$, so that in equation (32), the functions $\gamma(\phi)$ and $W(\phi)$ share the same angular dependence around the Fermi surface. For simplicity we assume the $d$-wave symmetry of the pseudogap as well as of the superconducting gap function

$$
W(\phi)=W \gamma(\phi),
$$

where $\gamma(\phi)=\sqrt{2} \cos (2 \phi)$ is the angular dependence of the gap around the Fermi surface [31].

After integrating over energy and carrying out the Matsubara sum, (Appendix A) we obtain the final equation for the critical temperature:

$$
\ln \frac{T_{c}}{T_{c 0}}=\int_{0}^{2 \pi} \frac{d \phi}{2 \pi} \gamma^{2}(\phi) \int_{-\infty}^{\infty} d x \Phi(x)\left\{\Psi\left(\frac{1}{2}\right)-\Psi\left(\frac{1}{2}+\frac{\Gamma+i x W(\phi)}{2 \pi T_{c}}\right)\right\},
$$

where the normalized distribution function $\Phi(x)$ can be written in terms of the modified Bessel function $K_{0}(x)$ as

$$
\Phi(x)=\frac{1}{\sqrt{2 \pi^{3}}} e^{-x^{2} / 4} K_{0}\left(\frac{x^{2}}{4}\right) .
$$

The relative factor of " $i$ " between the impurity and pseudogap term in this expression reflect the different mechanism by which impurity scattering and the pseudogap supress superconductivity. On the one hand, impurity scattering produces pair breaking, by scattering 
particles across the nodes of the order parameter. By contrast, the pseudogap suppresses superconductivity by removing states from around the Fermi energy.
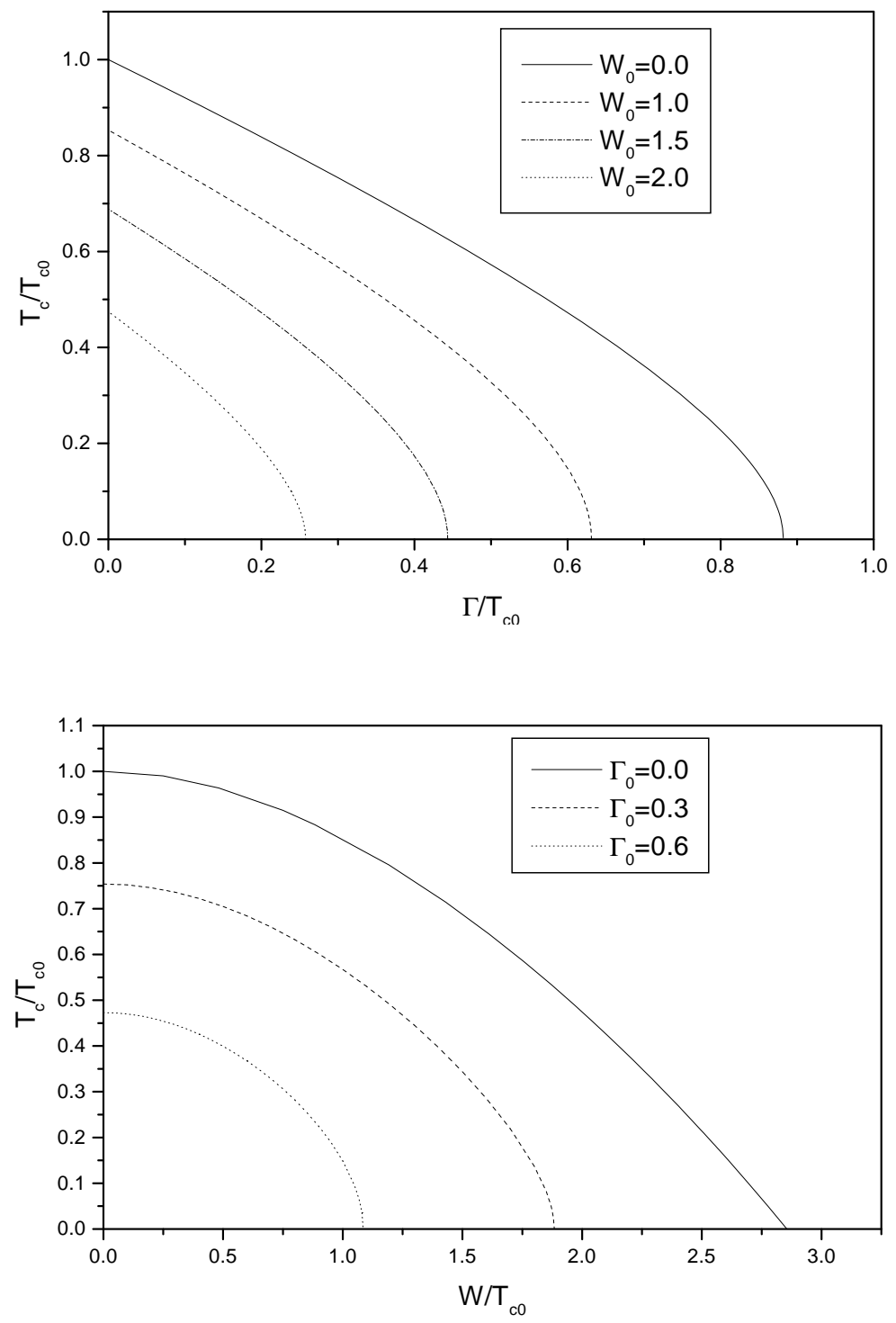

FIG. 3: (a) Critical temperature of a superconductor, normalized by the critical temperature of a pure superconductor without pseudogap $T_{c 0}$, vs impurity scattering $\Gamma$ in the presence of the pseudogap ( $W$ is an effective pseudogap width). (b) Critical temperature of a superconductor vs the effective width of the pseudogap for different values of impurity scattering rate $\Gamma$ (both $W$ and $\Gamma$ are normalized by $\left.T_{c 0}\right)$. These curves were all obtained from a numerical solution of equation (36). 
The theoretical results for the critical temperature dependence on the impurity scattering and the pseudogap, obtained by a numerical solution of Eq.(36), can be presented in different ways. Fig. 3a, for example, shows the variation of the transition temperature with impurity scattering rate for a set of different values of the pseudogap. In the limit where the pseudogap vanishes, Eq.(36) naturally reverts to the standard Abrikosov-Gorkov mean-field theory. Fig. 3b shows the dependence of the critical temperature on the pseudogap width for different ratios $\Gamma_{0} \equiv \Gamma / T_{c 0}$. These figures illustrate how both the pseudogap and impurity scattering suppress the superconductivity.

To illustrate this approach we now use it to develop a phenomenology for the cuprate phase diagram (Fig.4). We consider a model, in which the bare superconducting transition temperature is a linear function of doping $x$, vanishing at $x=0.3$ and the pseudogap is a rapidly reducing function of $x$, which vanishes at $x=0.19$. The effect of the pseudogap is to suppress superconductivity at low doping, leading to a maximum in the transition temperature in the vicinity of where the pseudogap goes to zero. When disorder is introduced the superconducting region is pushed to higher doping.

\section{FINITE CORRELATION LENGTH}

We now return to the problem of pseudo-gap formation in the presence of order parameter fluctuations with finite correlation length. This problem was originally considered by Lee

Rice and Anderson [1]. Following their approach, we consider Gaussian fluctuations of a static order parameter with finite correlation length and a real-space correlation function of the form

$$
<\Psi^{*}(x) \Psi\left(x^{\prime}\right)>=|\Psi|^{2} \exp \left(-\kappa\left|x-x^{\prime}\right|\right) e^{i 2 p_{F}\left(x-x^{\prime}\right)},
$$

where $|\Psi|^{2}$ is the mean square fluctuation of the order parameter field and $\kappa$ is the inverse correlation length.

Sadovskii [12, 13] later extended the FGM model by considering the effect of multiple scattering off order parameter fluctuations. The effect of superconducting pairing was incorporated into the model in recent papers [30]. In the case of infinite correlation length, the Sadovskii yields an essentially exact solution in one dimension. The Sadovskii approach is only approximate at finite correlation length [15]. For example, Bartosch and Kopietz [17] have shown, that the Dyson singularity in the density of states, which exists for any finite 


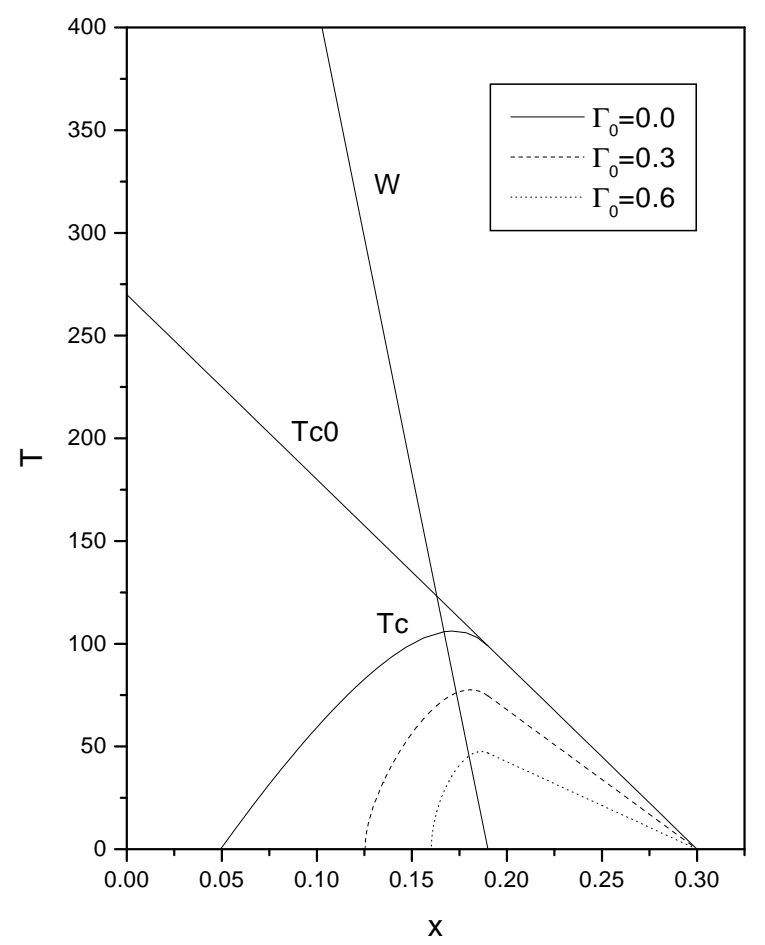

FIG. 4: Toy phase diagram of cuprates. Here $W$ is the effective energy scale of the pseudogap, for simplicity we assume that this quantity follows a linear dependence on doping $W=W_{\max }\left(1-x / x_{1}\right)$ $\left(x_{1} \simeq 0.19\right)$, while we let the critical temperature of a pure superconductor $T_{c 0}$ vanish at $x \simeq 0.3$, since in most experiments superconductivity is not observed for doping value exceeding $0.4 \div 0.5$. It is clearly seen now how the superconducting area shrinks while the scattering by normal impurities increases ( $\Gamma$ is impurity scattering rate $(34)$, normalized by $T_{c 0}$ ). This phase diagram is surprisingly similar to the figure 5 of experimental paper [29].

value of $\xi$, is missed by Sadovskii's algorithm. Nevertheless, the Sadovskii approach seems to be justified in 2D case for certain topologies of the Fermi surface [27]. Nevertheless, the Sadovskii approach appears to provide a remarkably good approximation to the density of states in a pseudogap system with finite correlation length [16, 28].

To overcome some of the difficulties encountered in these early treatments, Kopietz has recently suggested a simpler alternative model, which exhibits the same static spatial cor- 
relations originally considered by Lee Rice and Anderson, but which can be treated exactly. Kopietz's approach considers an order parameter of the form

$$
\Psi(x)=A e^{i q x}
$$

where the amplitude is a Gaussian random variable but the $q$ - vector is determined by a Lorentzian distribution, as follows

$$
P(\bar{A}, A, q)=\frac{1}{2 \pi^{2} W^{2}} e^{-\bar{A} A / 2 W^{2}} \frac{\kappa}{\left(\kappa^{2}+\left(q-Q_{o}\right)^{2}\right)}
$$

where we have chosen a one dimensional example, setting $Q_{o}=2 p_{F}$. The order parameter correlation function is then

$$
\left\langle\Psi^{*}(x) \Psi\left(x^{\prime}\right)\right\rangle=\int d A d \bar{A} d q P(\bar{A}, A, q) \bar{A} A e^{i q\left(x-x^{\prime}\right)}=W^{2} e^{-\kappa\left|x-x^{\prime}\right|} e^{i Q_{0}\left(x-x^{\prime}\right)} .
$$

For the commensurate case this recovers the form (37) first assumed by LRA.

The Hamiltonian which describes the coupling between the order parameter fluctuations and the electrons is given by

$$
H(\bar{A}, A, q)=\sum_{k} \epsilon_{k} c_{k}^{\dagger} c_{k}+\sum_{k}\left[A c_{k+q / 2}^{\dagger} c_{k-q / 2}+\bar{A} c_{k-q / 2}^{\dagger} c_{k+q / 2}\right]
$$

Unlike the scattering described in (9), here we have a quenched distribution of critical scattering $q$-vectors, and it is this feature that gives rise to the finite correlation length. By resuming all diagrams associated with scattering off the fluctuating field, Kopietz was able to derive the spectral density for this model.

We now show how the methods we have developed in the previous sections can be used to directly derive the Free energy for this model, from which secondary properties, such as the spectral Green function can also be derived. The starting point for our discussion is the Free energy in a given static configuration of the order parameter, given by

$$
F(\bar{A}, A, q)=-T \ln \operatorname{Tr}\left[e^{-\beta H(\bar{A}, A, q)}\right]
$$

Following our earlier discussion, the Gaussian fluctuations over the static classical fields are now represented by the quenched averaged Free energy

$$
F=\int d A d \bar{A} d q P(\bar{A}, A, q) F(\bar{A}, A, q)
$$


The integrals over $A, \bar{A}$ and $q$ can be carried out analytically (Appendix B) and yield the following result

$$
F=-2 T \int d x e^{-x} \operatorname{Tr} \ln \left[\left(\tilde{\omega}_{n}\right)^{2}+\left(\epsilon_{k+Q_{o} / 2}\right)^{2}+2 x W^{2}\right],
$$

where $\tilde{\omega}_{n}=\omega_{n}+\left(v_{F} \kappa / 2\right) \operatorname{sign} \omega_{n}$. Thus the most important effect of the finite correlation length is to introduce an imaginary scattering term inside the Greens function. Notice that the distribution over gap sizes has become exponential (Rayleigh), rather than Gaussian, as it was in the case where $Q_{o}=\pi$ : this is a consequence of having assumed an incommensurate $Q$ vector. Thus the essential physics of a finite correlation length manifests itself as an elastic impurity scattering potential.

Various extensions of these arguments are possible, such as the extension to two dimensions, the use of a commensurate scattering potential and the introduction of pairing terms, but we shall not pursue them in this paper.

\section{DISCUSSION AND CONCLUSIONS}

The central idea of this paper, was to point out that a large class of treatments of the pseudogap problem may be formulated in terms of a quenched average over a distribution of static classical order parameter fluctuations. We have illustrated this method, showing how it can be used to construct the Free energy of the pseudo-gap system, from which mean-field equations can be derived. The case of infinite correlation length is easier to handle, but a simplified version of the case with finite correlation length has also been treated.

The advantages of this method are that they permit us to begin with the Free energy functional, rather than working directly with Green functions. Thus we are able to introduce superconducting pairing into the formalism without having to reconsider a whole class of two-particle correlation functions [25].

Despite the utility of the new method, there are a number of important questions that arise as to its complete validity. There are two obvious weaknesses in the current approach

- The method ignores any frequency dependence in the fluctuations. For this reason, the method is only applicable at high temperatures.

- A secondary weakness is the assumption that the classical modes are strictly noninteracting. With this widely used assumption, we have shown that the fluctuations 
behave as a source of quenched disorder. The equivalence between classical fluctuations and quenched disorder only holds when the modes are non-interacting and classical

Both weaknesses are clearly issues of time scales. So long as the characteristic time-scale of the electrons is longer than the characteristic time scale of the fluctuations, then it is reasonable to treat them in the way we have outlined. If by contrast, we are interested in situations where the characteristic electron time scales become very large, such as the vicinity to a quantum critical point, then we might expect the methods used here to become invalid.

Nevertheless, there are clearly a wide number of applications for this approach. One of the interesting possible applications are transport properties of a pseudogap system, and these are currently under active investigation.

Acknowledgments We would like to thank J.O. Indekeu for early discussions on this work. Related discussions with P. Chandra, T. Giamarchi, E. Kuchinskii and especially M.V. Sadovskii are also gratefully acknowledged. This work is supported in part by the National Science Foundation grant nsf-dmr 9983156 (PC). The hospitality of LVSM, KULeuven and the MPICPS, Dresden, Germany where parts of this work were carried out, is highly appreciated.

\section{APPENDIX A}

The purpose of this appendix is to carry out the various integrations inside the gap equation

$$
\frac{1}{g}=2 T_{c} \int_{-\infty}^{\infty} d \zeta P(\zeta)\left\{\sum_{n, \kappa \in \frac{1}{2} \mathrm{BZ}} \frac{\gamma_{\kappa}^{2}}{\omega_{n}^{2}+\epsilon_{\kappa}^{2}+\zeta^{2} W_{\kappa-Q / 2}^{2}}\right\}
$$

where we have denoted

$$
P(\zeta)=\frac{1}{\sqrt{2 \pi}} e^{-\zeta^{2} / 2},
$$

If the pairing is dominated by processes near the Fermi energy, we may replace the momentum sum by an energy integral, thus

$$
\sum_{\vec{k}} \rightarrow N(0) \int \frac{d \theta}{2 \pi} \int_{-\infty}^{\infty} d \epsilon
$$


so the gap equation becomes

$$
\frac{1}{g}=2 \pi N(0) T_{c} \int_{-\infty}^{\infty} d \zeta P(\zeta) \int \frac{d \theta}{2 \pi} \gamma(\theta)^{2}\left\{\sum_{n=0}^{N_{\max }-1} \frac{1}{\sqrt{\tilde{\omega}_{n}^{2}+\zeta^{2} W(\theta)^{2}}}\right\} .
$$

where we have introduced an upper cutoff $N_{\max }=D / 2 \pi T_{c}$ into the Matsubara sum. To convert the Matsubara sum into a contour integral, we need to identify the function $G(z)$ that has poles at $z=i \tilde{\omega}_{n},\left(n \in\left\{0, N_{\max }-1\right\}\right)$. By using the identity

$$
\psi(z)=-C+\sum_{n=0}^{\infty}\left(\frac{1}{1+n}-\frac{1}{z+n}\right)
$$

we see that

$$
\sum_{n=0}^{N_{\max }^{-1}} \frac{1}{z+n}=\psi\left(z+N_{\max }\right)-\psi(z)
$$

so that

$$
G(z)=-2 \pi i T \sum_{n=0}^{N_{\max }} \frac{1}{z-i \tilde{\omega}_{n}}=\psi\left(\frac{1}{2}+\frac{D+\Gamma+i z}{2 \pi T}\right)-\psi\left(\frac{1}{2}+\frac{\Gamma+i z}{2 \pi T}\right)
$$

has the required properties. With this result, we can rewrite the Matsubara sum as

$$
\begin{aligned}
2 \pi T_{c} \sum_{n=0}^{N_{\max }} \frac{1}{\sqrt{\tilde{\omega}_{n}^{2}+\zeta^{2} W_{\theta}^{2}}} & =2 \pi T_{c} \int \frac{d z}{2 \pi i}\left(\frac{i}{\sqrt{z^{2}-\zeta^{2} W_{\theta}^{2}}}\right)[i G(z)] \\
& =\int \frac{d z}{2 \pi} \frac{i}{\sqrt{z^{2}-\zeta^{2} W_{\theta}^{2}}} G(z)
\end{aligned}
$$

where the integral is taken anticlockwise around the positive imaginary axis. Notice that we have analytically extended $i \omega_{n} \rightarrow z$ by replacing $\sqrt{\tilde{\omega}_{n}^{2}+\zeta^{2} W_{\theta}^{2}} \rightarrow-i \sqrt{z^{2}-\zeta^{2} W_{\theta}^{2}}$. With this choice, there is a branch-cut running from $z=-\zeta W_{\theta}$ to $z=\zeta W_{\theta}$. Above and below the branch-cut, $\sqrt{(x \pm i \delta)^{2}-\zeta^{2} W_{\theta}^{2}}= \pm i \sqrt{\zeta^{2} W_{\theta}^{2}-x^{2}}$. We may now distort the contour so that it runs clockwise around this branch-cut, to obtain

$$
2 \pi T \sum_{n=0}^{N_{\max }} \frac{1}{\sqrt{\tilde{\omega}_{n}^{2}+\zeta^{2} W^{2}}}=\int_{-\zeta W_{\theta}}^{\zeta W_{\theta}} \frac{d x}{\pi} \frac{1}{\sqrt{\zeta^{2} W_{\theta}^{2}-x^{2}}} G[x] .
$$

Along the branch-cut, we may replace

$$
\psi\left(\frac{1}{2}+\frac{D+\Gamma+i z}{2 \pi T}\right) \rightarrow \ln \left(\frac{D}{2 \pi T}\right)
$$

so that inside the integral we can use

$$
G[x]=\ln \left(\frac{D}{2 \pi T}\right)-\psi\left(\frac{1}{2}+\frac{\Gamma+i x}{2 \pi T}\right)
$$




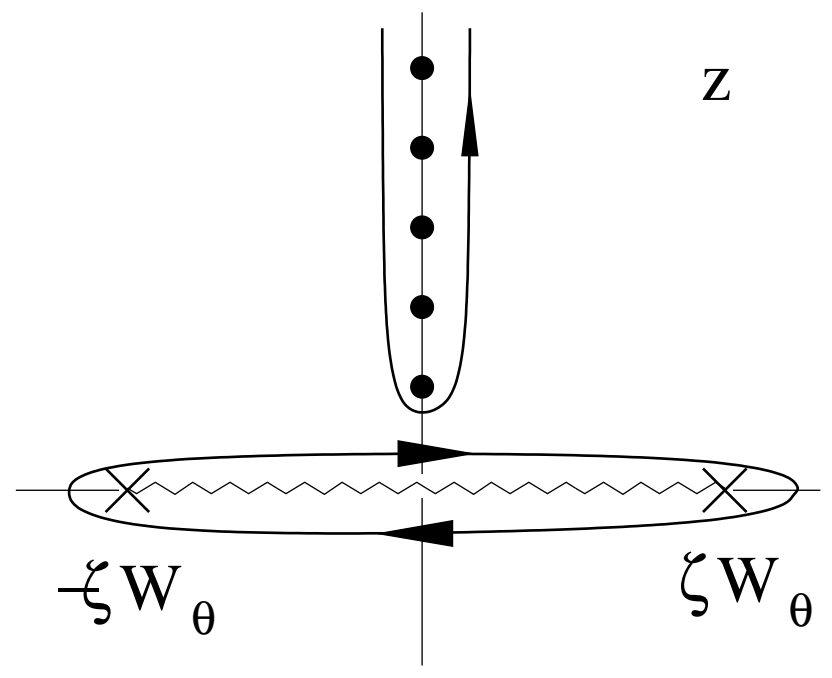

FIG. 5: Showing how contour around the poles of $G(z)$ on the positive imaginary axis in equation (49) is distorted around the branch cut running between $\pm \zeta W_{\theta}$.

At this point, the gap equation takes the form

$$
\frac{1}{g N(0)}=\int \frac{d \theta}{2 \pi} \gamma(\theta)^{2} \int_{-\infty}^{\infty} d \zeta P(\zeta)\left\{\int_{-\zeta W_{\theta}}^{\zeta W_{\theta}} \frac{d x}{\pi} \frac{1}{\sqrt{\zeta^{2} W_{\theta}^{2}-x^{2}}} G[x]\right\} .
$$

We can further simplify this integral by reversing the order of innermost $\zeta$ and $x$ integrations. We do this as follows

$$
I=\int_{-\infty}^{\infty} \frac{d \zeta}{\sqrt{2 \pi}} e^{-\zeta^{2} / 2} \int_{-\zeta W_{\theta}}^{\zeta W_{\theta}} \frac{d x}{\pi}[\ldots]=\sqrt{\frac{2}{\pi^{3}}} \int_{-\infty}^{\infty} d x \int_{\frac{|x|}{W_{\theta}}}^{\infty} d \zeta e^{-\zeta^{2} / 2}[\ldots]
$$

Next we make the change of variable, $u=\frac{\zeta^{2}}{2}-\frac{x^{2}}{2 W_{\theta}^{2}}$, so that the inner integral becomes

$$
I=\sqrt{\frac{2}{\pi^{3}}} \int_{-\infty}^{\infty} d x e^{-x^{2} / 2 W_{\theta}^{2}} \int_{0}^{\infty} d u \frac{e^{-u}}{\sqrt{2\left(u+x^{2} / 2 W_{\theta}^{2}\right)}}[\ldots]
$$

Replacing the argument inside the integral by

$$
[\ldots]=\frac{G[x]}{\sqrt{\zeta^{2} W_{\theta}^{2}-x^{2}}}=\frac{G[x]}{W_{\theta} \sqrt{2 u}}
$$

we obtain

$$
I=\frac{1}{\sqrt{2 \pi^{3}}} \int_{-\infty}^{\infty} \frac{d x}{W_{\theta}} e^{-x^{2} / 2 W_{\theta}^{2}} G[x] \int_{0}^{\infty} d u \frac{e^{-u}}{\sqrt{u\left(u+x^{2} / 2 W_{\theta}^{2}\right)}}
$$




$$
=\frac{1}{\sqrt{2 \pi^{3}}} \int_{-\infty}^{\infty} d x e^{-x^{2} / 2} G\left(x W_{\theta}\right) \int_{0}^{\infty} d u \frac{e^{-u}}{\sqrt{u\left(u+x^{2} / 2\right)}}
$$

The integral on the right hand side can be carried out analytically,

$$
\int_{0}^{\infty} d u \frac{e^{-u}}{\sqrt{u(u+t)}}=e^{t / 2} K_{0}\left(\frac{t}{2}\right)
$$

where $K_{0}(x)$ is the modified Bessel function $\left(y=K_{0}(x)\right.$ is the solution to the differential equation $\left.x^{2} y+x y^{\prime}-x^{2} y=0\right)$, so that

$$
I=\int_{-\infty}^{\infty} d x \Phi(x) G\left(x W_{\theta}\right)
$$

where

$$
\Phi(x)=\frac{1}{\sqrt{2 \pi^{3}}} e^{-x^{2} / 4} K_{0}\left(x^{2} / 4\right)
$$

is a normalized distribution function $\left(\int_{-\infty}^{\infty} d x \Phi(x)=1\right)$. Using this to replace the inner two integrals of the gap equation (52) we obtain

$$
\begin{aligned}
\frac{1}{g N(0)} & =\int \frac{d \theta}{2 \pi} \gamma(\theta)^{2} \int_{-\infty}^{\infty} d x \Phi(x)\left[\ln \left(\frac{D}{2 \pi T}\right)-\psi\left(\frac{1}{2}+\frac{\Gamma+i W_{\theta} x}{2 \pi T}\right)\right] \\
& =\ln \left(\frac{D}{2 \pi T_{c}}\right)-\int \frac{d \theta}{2 \pi} \gamma(\theta)^{2} \int_{-\infty}^{\infty} d x \Phi(x) \psi\left(\frac{1}{2}+\frac{\Gamma+i W_{\theta} x}{2 \pi T}\right)
\end{aligned}
$$

where we have used the normalization of the distribution function and $\gamma(\theta)^{2}$ to extract the logarithm from the integral. If we set $W=0$ and $\Gamma=0$ in this expression, we obtain the familiar result

$$
\frac{1}{g N(0)}=\ln \left(\frac{D}{2 \pi T_{c 0}}\right)-\psi\left(\frac{1}{2}\right)
$$

enabling us to eliminate the coupling constant from the gap equation, writing

$$
\ln \left(\frac{T_{c}}{T_{c 0}}\right)=\int \frac{d \theta}{2 \pi} \gamma(\theta)^{2} \int_{-\infty}^{\infty} d x \Phi(x)\left[\psi\left(\frac{1}{2}\right)-\psi\left(\frac{1}{2}+\frac{\Gamma+i W_{\theta} x}{2 \pi T}\right)\right]
$$

\section{APPENDIX B}

The purpose of this section is to average the Free energy over the classical fields of the pseudogap. If we write the electron field in a two-component notation as

$$
C_{k}=\left(\begin{array}{c}
c_{k+q / 2} \\
c_{k-q / 2}
\end{array}\right)
$$


then in the presence of the scattering potential

$$
H_{I}=\sum_{k}\left[A c_{k+q / 2}^{\dagger} c_{k-q / 2}+\bar{A} c_{k-q / 2}^{\dagger} c_{k+q / 2}\right]
$$

the corresponding electron Greens function is given by

$$
\begin{aligned}
-\left\langle C_{k \alpha}(\tau) C_{k \beta}^{\dagger}(0)\right\rangle & =\mathcal{G}_{\alpha \beta}(k, \tau) \\
\mathcal{G}_{\alpha \beta}(k, \tau) & =T \sum_{n} \mathcal{G}\left(k, i \omega_{n}\right) e^{-i \omega_{n} \tau}
\end{aligned}
$$

where

$$
\mathcal{G}\left(\vec{k}, i \omega_{n}\right)=\left[i \omega_{n}-\epsilon_{k+(q / 2) \tau_{3}}-A \tau_{-}-\bar{A} \tau_{+}\right]^{-1}
$$

is the inverse propagator of the electron in the pseudogap field. To obtain this expression we have kept scattering between states with $k \sim \pm k_{F}$, neglecting scattering into high energy states with $k \sim \pm\left(k_{F}+n q\right)$. The free energy is then given by

$$
F=\int \frac{d A d \bar{A}}{2 \pi \Delta^{2}} e^{-\bar{A} A / 2 W^{2}} \int d q \frac{1}{\pi\left(\kappa^{2}+\left(q-Q_{o}\right)^{2}\right)} F[q, \bar{A}, A]
$$

where

$$
F[q, \bar{A}, A]=-T \operatorname{Tr} \ln \left[-\mathcal{G}^{-1}\left(k, i \omega_{n}\right)\right]
$$

To carry out the Lorentzian integral over q, we first linearize the electron kinetic energy around the wavevector $q=Q_{o}$, writing

$$
\begin{aligned}
& -T \int \frac{d q}{\pi} \frac{\kappa}{\left(q-Q_{0}\right)^{2}+\kappa^{2}} \operatorname{Tr} \ln \left[i \omega_{n}-\epsilon_{k+\left(Q_{o} / 2\right) \tau_{3}}-v_{F}\left(q-Q_{0}\right)-A \tau_{-}-\bar{A} \tau_{+}\right] \\
= & -T \int \frac{d q}{\pi} \frac{\kappa}{q^{2}+\kappa^{2}} \operatorname{Tr} \ln \left[i \omega_{n}-\epsilon_{k+\left(Q_{o} / 2\right) \tau_{3}}-v_{F} q-A \tau_{-}-\bar{A} \tau_{+}\right]
\end{aligned}
$$

This Lorentzian momentum integral has two poles in the complex plane at $q-Q_{o}= \pm i \kappa$. For $\omega_{n}>0$, the poles of the logarithm are in the upper half complex plane, so we complete the contour in the lower half plane, picking up the sole contribution to the pole from $q=-i \kappa$. For the opposite sign, i.e. $\omega_{n}<0$ we complete the contour in the upper half plane. The result of this procedure is

$$
\begin{aligned}
F & =-T \int \frac{d A d \bar{A}}{2 \pi \Delta^{2}} e^{-\bar{A} A / 2 \Delta^{2}} \operatorname{Tr} \ln \left[i \tilde{\omega}_{n}-\epsilon_{k+\left(Q_{o} / 2\right) \tau_{3}}-A \tau_{-}-\bar{A} \tau_{+}\right] \\
\tilde{\omega}_{n} & =\omega_{n}+\left(v_{F} \kappa / 2\right) \operatorname{sign}\left(\omega_{n}\right)
\end{aligned}
$$


showing that the effect of the finite correlation length is to introduce a damping term into the propagator. We can further simplify this result by writing the the nesting condition $\epsilon_{k+Q_{o} / 2}=-\epsilon_{k-Q_{o} / 2}$ as $\epsilon_{k+Q_{o} / 2 \tau_{3}}=\epsilon_{k-Q_{o} / 2} \tau_{3}$, so that

$$
\begin{aligned}
F & =-T \int \frac{d A d \bar{A}}{2 \pi \Delta^{2}} e^{-\bar{A} A / 2 \Delta^{2}} \operatorname{Tr} \ln \left[i \tilde{\omega}_{n}-\epsilon_{k+Q_{o} / 2} \tau_{3}-A \tau_{-}-\bar{A} \tau_{+}\right], \\
& =-2 T \int \frac{d A d \bar{A}}{2 \pi \Delta^{2}} e^{-\bar{A} A / 2 \Delta^{2}} \operatorname{Tr} \ln \left[\left(\tilde{\omega}_{n}\right)^{2}+\left(\epsilon_{k+Q_{o} / 2}\right)^{2}+\bar{A} A\right],
\end{aligned}
$$

To simplify the Gaussian integral over the pseudo gap amplitude, we simply make the change of variables

$$
\begin{aligned}
& A=\sqrt{2 x} W e^{i \theta} \\
& \bar{A}=\sqrt{2 x} W e^{-i \theta}
\end{aligned}
$$

so that

$$
\frac{d A d \bar{A}}{2 \pi W^{2}} e^{-\bar{A} A / 2 W^{2}}=\frac{d x d \theta}{2 \pi} e^{-x}
$$

so that the Free energy becomes

$$
F=-2 T \int d x e^{-x} \operatorname{Tr} \ln \left[\left(\tilde{\omega}_{n}\right)^{2}+\left(\epsilon_{k+Q_{o} / 2}\right)^{2}+2 x W^{2}\right],
$$

introducing a Rayleigh distribution of gap sizes.

[1] P. Lee, M. Rice and P. W. Anderson, Phys. Rev. Lett. 31, 462 (1973).

[2] M. Randeria, Varenna Lectures 1997, preprint cond-mat/9710223 M. Randeria, J. C. Campuzano, Varenna Lectures 1997, preprint cond-mat/9709107.

[3] T. Timusk, B. Statt, Rep. Prog. Phys. 62, 61 (1999).

[4] M.V. Sadovskii, Usp. Fiz. Nauk 171, 539 (2001); (Phys. Uspekhi 44, 515 (2001)).

[5] A. P. Kampf, J. R. Schrieffer, Phys. Rev. B 41, 6399 (1990), 42, 7967(1990); V. Barzykin, D. Pines, Phys. Rev. B 52,13585(1995); A. Chubukov, D. Pines, B. Stojkovic, J. Phys. Condensed Matter 8, 10017 (1996).

[6] V. J. Emery (BNL), S. A. Kivelson (UCLA) and J. M. Tranquada (BNL), Proc. Natl. Acad. Sci. USA 96, 8814 (1999) and references therein.

[7] V. B. Geshkenbein, L. B. Ioffe, A. I. Larkin, Phys. Rev. B 55, 3173 (1997), V. Emery, S. A. Kivelson, O. Zachar, Phys. Rev. B 56, 6120 (1997). 
[8] G. Grüner, "Density Waves in Solids", Addison Wesley (1994) and references therein.

[9] A. Yeh, Yeong-Ah Soh, J. Brooke, G. Aeppli, T. F. Rosenbaum and S. M. Hayden, Nature, in press (2002).

[10] D. S. Dessau et al., J. Electron Spectrosc. Rel. Phenom. 117-118, 265 (2001).

[11] Y. S. Lee et al., Phys. Rev. B 66, 041104(R) (2002), T. W. Noh et al., Physica C 364-365, $480(2001)$.

[12] M.V. Sadovskii, Zh. Eksp. Teor. Fiz. 66, 1720 (1974) [Sov. Phys. JETP 39, 845 (1974)].

[13] M. V. Sadovskii, Zh. Eksp. Teor. Fiz. 77, 2070 (1979) [Sov. Phys. JETP bf 50, 989 (1979)].

[14] J. Schmalian, D. Pines, B. Stojkovic, Phys. Rev. Lett. 80, 3839 (1998); Phys. Rev. B 60, 667 (1999).

[15] O. Tchernyshyov, Phys. Rev. B 59, 1358 (1999).

[16] A. Millis and H. Monien, Phys. Rev. B 61, 12496 (2000).

[17] L. Bartosch, P. Kopietz, Phys. Rev. B 60, 15488 (1999).

[18] J. L. Tallon et al., Phys. Rev. Lett. 79, 5294 (1997), J. L. Tallon, Phys. Rev. B 58, R5956 (1998).

[19] L. Bartosch, P. Kopietz, Eur. Phys. J. B 17, 555 (2000).

[20] H. Monien, Phys. Rev. Lett. 87, 126402 (2001).

[21] P. G. De Gennes and J. Friedel, J. Phys. Chem Solids 4, 71 (1958).

[22] M. E. Fisher and J. S. Langer, Phys. Rev. Lett., 20, 665 (1968).

[23] P. Chandra, J. Phys. Condens Matter 50, 10067-10080, (1989).

[24] P. Monthoux, Phil. Mag B. 79, 15 (1999).

[25] A. Posazhennikova, M. V. Sadovskii, JETP 115, 632 (1999).

[26] S. F. Edwards, P. W. Anderson, J. Phys. F5, 965 (1975), also reference book D. Chowdhury "Spin glasses and other frustrated systems", World Scientific, 1986, Singapore.

[27] E. Z. Kuchinskii, M. V. Sadovskii, Zh. Eksp. Teor. Fiz. (JETP) 115, 1765 (1999).

[28] M. V. Sadovskii, Physica C 341-348, 811 (2000).

[29] S. H. Naqib, J. R. Cooper, J. L. Tallon, and C. Panagopoulos, cond-mat/0209457

[30] E. Z. Kuchinskii, M. V. Sadovskii, Zh. Eksp. Teor. Fiz. (JETP) 119, 553 (2001); E. Z. Kuchinskii, M. V. Sadovskii, Zh. Eksp. Teor. Fiz. (JETP) 117, 613 (2000); Physica C 341-348, 879 (2000) .

[31] Another way to introduce the pseudogap is to assume the appearance of so-called "hot" regions 
on the Fermi surface with non-zero pseudogap [25] 